\title{
Activation induced cytidine deaminase mutant (AID-His130Pro) from Hyper IgM 2 patient retained mutagenic activity on SHM artificial substrate
}

Hanen Ouadani $^{\mathrm{a}-\mathrm{b}}$, Imen Ben-Mustapha ${ }^{\mathrm{a}-\mathrm{b}}$, Meriem Ben-ali ${ }^{\mathrm{a}-\mathrm{b}}$ Beya Larguèche $^{\mathrm{a}}$, Tihana Jovanic ${ }^{c}$ 1, Sylvie Garcia', Benoit Arcangioli', Houda Elloumi-Zghal ${ }^{\mathrm{a}}$ 2, Dahmani Fathallah $^{\mathrm{a}}$, Mongia Hachicha ${ }^{\mathrm{f}}$, Hatem Masmoudi ${ }^{\mathrm{g}}$, François Rougeon ${ }^{\mathrm{c}}$, MohamedRidha Barbouche $\mathrm{a}^{\mathrm{a}-\mathrm{b} *}$

${ }^{a}$ Laboratory of Transmission, Control and Immunobiology of Infection (LR11IPT02), Institut Pasteur de Tunis.

${ }^{\mathrm{b}}$ University Tunis El Manar, Tunis, Tunisia.

${ }^{\mathrm{c}}$ Biochemistry and Genetics Development Unit (URA CNRS 2581), Institut Pasteur Paris, France.

${ }^{\mathrm{d}}$ Laboratory of Lymphocyte Population Biology, Institut Pasteur Paris, France.

${ }^{\mathrm{e}}$ Laboratory of Dynamics of the Genome, Institut Pasteur, France.

${ }^{\mathrm{f}}$ Department of Pediatrics, Hedi Chaker Hospital, Sfax, Tunisia.

${ }^{g}$ Laboratory of Immunology, Habib Bourguiba Hospital, Sfax, Tunisia.

* Correspondence to: Mohamed-Ridha Barbouche, Laboratory of Transmission, Control and Immunobiology of Infection (LR11IPT02), Institut Pasteur de Tunis, 13 Place Pasteur-BP74, 1002 TunisBelvedere, Tunisia. Phone number: (+216) 71843 317. E-mail: ridha.barbouche@pasteur.rns.tn 


\begin{abstract}
Activation induced cytidine deaminase (AID) is an essential enzyme for class switch recombination (CSR) and somatic hypermutation (SHM) during the secondary immune response. Mutations in the AICDA gene are responsible for Hyper IgM 2 (HIGM2) syndrome where both CSR and SHM or only CSR are affected. Indeed, triggering either of the two mechanisms requires the DNA deamination activity of AID. Besides, different domains of AID may be differentially involved in CSR and SHM through their interaction with specific cofactors. Herein, we studied the AID-induced SHM activity of the AID-His130Pro mutant identified in a patient with HIGM2 syndrome. AID mutagenic activity was monitored by the reversion of nonsense mutations of the EGFP gene assessed by flow cytometry. We found that the His130Pro mutation, which affects CSR, preserves AID mutagenic activity. Indeed, the His130 residue is located in a putative specific CSR region in the APOBEC-like domain, known to involve CSR specific cofactors that probably play a major role in AID physiological activities.
\end{abstract}

\title{
Key words:
}

Activation induced cytidine deaminase, class switch recombination, somatic hypermutation, AID mutagenic activity, cofactors, Hyper IgM 2 


\section{Introduction}

Class Switch Recombination (CSR) and Somatic Hypermutation (SHM) are the mechanisms that allow antibodies to ensure diverse effector functions and to reach affinity maturation. CSR occurs by intrachromosomal deletional recombination within Switch (S) regions located upstream of constant heavy chain of immunoglobulins (Ig) genes (except $\mathrm{C} \delta$ ) and leads to production of $\operatorname{Ig}$ isotypes other than $\operatorname{IgM}(\mathrm{Xu}$ et al., 2012). SHM results mainly from single nucleotide substitutions within the variable (V) genes of immunoglobulins (Peled et al., 2008). These two mechanisms are initiated by the enzyme Activation Induced Cytidine Deaminase (AID) following interaction between CD40 and CD40L expressed on B cells and activated $\mathrm{T}$ cells respectively (Crouch et al., 2007; Muramatsu et al., 2000). Indeed, genetic defects of AID underlie Hyper IgM 2 (HIGM2) syndrome (Durandy et al., 2013; Muramatsu et al., 2000; Revy et al., 2000). AID is a protein of $24 \mathrm{kD}$ encoded by AICDA gene and specifically expressed in activated B cells present within germinal centers of secondary lymphoid organs (Muto et al., 2000). Because of its mutagenic activity, AID expression and activity are tightly regulated at transcriptional and post-translational levels (Crouch et al., 2007; Zan and Casali, 2013). The cellular localization of AID is regulated by nuclear export signal (NES) and nuclear localization signals (NLS) which control the shuttling of the protein between the cytoplasm and the nucleus in an active manner (Ito et al., 2004; Patenaude et al., 2009). The catalytic domain of AID is common to the cytidine deaminase family. It includes a motif (His-X-Glu-..-Pro-Cys-X-X-Cys) involved in zinc coordination where His56, Cys87 and Cys90 bind $\mathrm{Zn}^{2+}$ and the carboxylic acid group of Glu58 serves as a general acid-base catalyst involved in DNA deamination (Wedekind et al., 2003). AID also shares an APOBEC-like domain with 
the cytidine deaminase family. This domain contain the hotspot recognition loop which contributes to the differential recognition of specific DNA sequence motifs (Kohli et al., 2009). How AID can target CSR and SHM in different ways put out the hypothesis of the presence of specific cofactors. Indeed, AID has been proposed to be a docking protein acting by the recruitment of specific cofactors which mediate either mechanism through interaction with different AID domains (Durandy et al., 2006; Shinkura et al., 2004). Analyses of AID mutant proteins activity in HIGM2 patients allow a better understanding of CSR and SHM targeting mechanism. Today, 45 mutations of the AICDA gene are reported (Caratão et al., 2013; Mahdaviani et al., 2012; Ouadani et al., 2015; Patiroglu et al., 2015; Trotta et al., 2016) and are mainly homozygous or compound heterozygous with an autosomal recessive inheritance, except two heterozygous mutations with a dominant negative effect (Imai et al., 2005; Kracker et al., 2010). Most of them lead to the loss of both CSR and SHM activities. Nevertheless, AID C-terminal mutations display a defect in CSR with normal SHM activity showing that this domain is CSR specific (Durandy et al., 2006). Indeed, the AID C-terminal domain is required for $\mathrm{S}$ region recombination (Sabouri et al., 2014). Interestingly, AID Arg112Cys mutation located outside the AID C-terminal domain seems to retain residual SHM activity (Mahdaviani et al., 2012; Mu et al., 2012). Otherwise, analysis of AID mutants generated by site-directed mutagenesis show that some AID N-terminal mutations retain CSR activity but lose SHM (Shinkura et al., 2004). Recently, we have identified a novel AID mutation (His130Pro), in HIGM 2 patients, located in the APOBEC-like domain (Ouadani et al., 2015). Fascinatingly, the His 130 residue belongs to a putative RNA-binding region that would likely be CSR specific, through the interaction of AID with specific RNA (Mondal et al., 2016; Zheng et al., 2015) and 
might not affect SHM. We aim herein to assess the SHM activity of AID-His130Pro mutant using an in vitro mutagenesis assay on an artificial SHM substrate already developed by Rougeon and collaborators (Jovanic et al., 2008).

\section{Materials and Methods}

\subsection{Ethical note}

Written consent was obtained before studies and adherence to the Declaration of Helsinki Principles was maintained.

\subsection{AID protein analysis}

EBV-lymphoblastoide cell lines (LCLs) were lysed for $5 \mathrm{~min}$ in $12.5 \mathrm{mM}$ Tris $\mathrm{HCl}, 4 \%$ Glycerol and 0.4\% SDS. Proteins were quantified using Bicinchoninic Acid Protein Assay Kit (Sigma), subjected to $15 \%$ SDS PAGE polyacrylamid gel and transferred to a PolyVinyliDene Fluoride (PVDF) membrane. AID and $\beta$-actin were revealed using respectively a rabbit monoclonal anti AID (Cell Signaling) and a rabbit monoclonal anti actin (Santa Cruz Biotechnology). Western blots were analyzed by enhanced chemiluminescence with Pierce ECL Western blotting reagent (Thermo Scientific).

The functional impact of the His130Pro mutation was predicted with the PolyPhen-2 (Adzhubei et al., 2010) and Sorts Intolerant From Tolerant Substitutions (SIFT) (Kumar et al., 2009) algorithms. These methods consider protein structure and/or sequence conservation information for each gene.

\subsection{Plasmid constructs and Site-Directed Mutagenesis}


Wild type (AID-wt) and mutant (AID-His130Pro) AICDA cDNA (accession n. NM_020661) were amplified from EBV-LCLs using the following specific primers: Forward (5'GGGTTTGCTAGCATGGACAGCCTCTT3'); Reverse (5' TACCCTCTCGAGCTATCAAAGTCCCA3'). PCR products were immediately cloned using TA cloning Kit (Invitrogen) following the manufacturer's specifications. The recombinant plasmids were purified from Escherichia coli XL1 using Wisard Mini prep Kit (Promega) and then sequenced using vector specific primers (M13 Forward 5'GTAAAACGACGGCCAG-3'， M13 Reverse 5'-CAGGAAACAGCTATGAC-3'). DNA fragments were liberated from PCRII Topo vector using XhoI and NheI (Roche) restriction enzymes then ligated into XhoI-NheI digested pCINeo vector using T4 ligase (Roche). The recombinant plasmids were purified from Escherichia coli XL1 competent cells using Midi prep kit (Qiagen).

The recombinant pCINeo-AID-His56Tyr, in which the mutated AID gene contain the c.166T $>C$ mutation, was generated from the recombinant pCINeo-AID-wt plasmid with the QuikChange site directed mutagenesis system (Stratagene, La Jolla, CA), according to the manufacturer's instructions, using the following primers: Forward (5' GCAATAAGAACGGTGCTACGTGGAATTGCTCTTC3') and Reverse (5'GAAGAGCAATTCCACGTAGCAGCCGTTCTTATTGC3'). This mutant was used as a negative control since the p.(His56Tyr) alters both CSR and SHM (Durandy et al., 2006).

\subsection{Jurkat stable cell lines establishment}

Jurkat cells were cultured in RPMI glutamax with $10 \%$ FBS, $100 \mathrm{U} / \mathrm{ml}$ penicillin and $100 \mathrm{~g} / \mathrm{ml}$ streptomycin at $37^{\circ} \mathrm{C}$ and $5 \% \mathrm{CO}_{2}$. Stable Jurkat cell lines, expressing AID-wt 
(Jurkat AID-wt), AID-His130Pro (Jurkat AID-His130Pro) and AID-His56Tyr (Jurkat AID-His56Tyr), were obtained by nucleofection of $10^{6}$ Jurkat cells with $2 \mu \mathrm{g}$ of the corresponding recombinant pCINeo plasmids according to the manufacturer's instructions (Amaxa ${ }^{\mathrm{TM}}$ 4D-Nucleofector ${ }^{\mathrm{TM}}$ Protocol for Jurkat clone E6.1 from Lonza). Forty eight hours after transfection, selection was made by addition of Neomycin (Sigma-Aldrich) at a final concentration of $2 \mathrm{mg} / \mathrm{ml}$. Medium was changed every 2-3 days until all cells of the negative control (non-transfected Jurkat cells) were dead. Cells were then amplified by batch culture in RPMI glutamax with $10 \%$ FBS, $100 \mathrm{U} / \mathrm{ml}$ penicillin, $100 \mathrm{~g} / \mathrm{ml}$ streptomycin and $2 \mathrm{mg} / \mathrm{ml}$ Neomycin, at $37^{\circ} \mathrm{C}$ and $5 \% \mathrm{CO}_{2}$. They were tested for AICDA gene integration and expression by reverse transcriptase (RT)PCR and sequencing. AID proteins expression was assessed by western blot as described previously.

\section{5. $\quad$ AID-induced mutagenesis analysis}

SHM vectors used in this assay are the wild type vector pEM7-EGFP, which express the EGFP protein, and the mutant pEM7-TAG182 vector which is unable to express the protein (Jovanic et al., 2008).

$2 \times 10^{6}$ of each Jurkat stable cell line were transfected with either $3 \mu \mathrm{g}$ of pEM7-EGFP or pEM7-TAG182 by nucleofection as described above. Twenty-four hours after transfection cells were centrifuged, washed by PBS, re-suspended in PBS with $0.5 \%$ SVF and analyzed by flow cytometry.

\section{Results}

\subsection{AID-His130Pro mutant characteristics}


The His130Pro nonsynonymous mutation was predicted to be benign by both PolyPhen2 and SIFT algorithms suggesting that the His130Pro mutation does not affect drastically the protein structure. On the other hand, western blot analysis of EBV LCLs lysates from HIGM2 patient, carrying the His130Pro mutation, revealed a low expression level of the mutant AID-His130Pro protein compared to the wild type. This indicates that this mutation does not affect mRNA translation but alters the protein expression stability (Figure 1a).

\section{2. $\quad$ AID protein expression in Jurkat stable cell lines}

Since only a limited amount of patients' peripheral blood cells was available, we performed an in vitro SHM assay on established Jurkat stable cell lines, expressing either AID-wt or AID-His130Pro proteins. In addition, an SHM assay negative control, consisting in the Jurkat cell line expressing the AID-His56Tyr mutant generated by sitedirected mutagenesis, was used. Indeed, this mutant is known to alter both CSR and SHM (Durandy et al., 2006). The different Jurkat stable cell lines effectively expressed AID as demonstrated by RT-PCR analysis of wild type and mutant AID transcripts (Figure 1b). Western blot analysis of Jurkat cell lines lysates showed that both wild type and mutants AID proteins are expressed. As expected, AID-His130Pro protein expression was lower than AID-wt but AID-His56Tyr mutant expression was even lower than AID-His130Pro (Figure 1a).

\subsection{AID-induced mutagenesis analysis}

The assessment of the AID-induced mutagenesis was monitored by the reversion of the TAG182 nonsense mutation of the EGFP gene cloned in the pEM7-TAG182 vector. 
The reversion of the mutation restores fluorescence allowing the reversion rate to be evaluated by flow cytometry. The reversion rate is represented by the percentage of reverted fluorescent cells derived from transfection of Jurkat cells with pEM7-TAG182. This rate depends on the transfection efficiency of the concerned Jurkat cell line that is estimated by the percentage of fluorescent cells derived from transfection with the wild type pEM7-EGFP vector. Thus, we defined -as already reported- the mutagenic activity as the reversion rate relative to the transfection efficiency (Jovanic et al., 2008). Transfection efficiency was $77 \%$ for Jurkat cell line and around $62-64 \%$ for stable Jurkat cells expressing AID-wt, AID-His130Pro and AID-His56Tyr (Figure 2).

In two distinct experiences, AID-wt was able to revert the non-sense EGFP mutation as demonstrated by the percentage of the reverted fluorescent Jurkat AID-wt cells (4.4\% and $6.1 \%$, respectively) compared to the absence of reverted fluorescent Jurkat cells not expressing AID (Figure 3). Calculated as described above, the average of the AID-wt mutagenic activity from the two experiments was about $8 \%( \pm 2)$ (Figure 4).

In parallel, we assessed the mutagenic activity of the AID-His56Tyr mutant which is known to affect both CSR and SHM because the p.(His56Tyr) mutation alters the AID catalytic domain (Durandy et al., 2006; Ta et al., 2003). As expected, the mutagenic activity of this mutant was absent since the percentage of reverted fluorescent Jurkat AID-His56Tyr cells did not exceed $0.5 \%$ (Figure 3).

Interestingly, the Jurkat AID-His130Pro cell line showed significant reversion rates, which were $3.6 \%$ and $2.03 \%$, respectively (Figure 3), bringing the mutagenic activity to $5 \%( \pm 2 \%)$ (Figure 4). This indicates that the AID-His130Pro mutant is able to revert the non-sense EGFP mutation meaning it preserves its mutagenic activity.

\section{Discussion}


We already reported a novel AID mutation (His130Pro) in five Tunisian HIGM2 patients. This mutation, located in the APOBEC-like domain, alters CSR mechanism as supported by presence of high IgM levels and absent $\operatorname{IgG}$ and $\operatorname{IgA}$ in these patients (Ouadani et al., 2015). According to PolyPhen-2 and SIFT algorithms prediction, the His130Pro does not alter AID protein structure. Nevertheless, western blot analysis indicates that it may affect the protein expression stability. Unfortunately, there are no available data explaining the involvement of His130 residue in AID stability (Le and Maizels, 2015; Mu et al., 2012; Prochnow et al., 2007). Several mutations have been reported in the APOBEC like domain of AID. Some of these mutants retained deamination activity despite altered CSR (Mahdaviani et al., 2012; Zhu et al., 2003). Intriguingly, Shivarof and his collaborators provided evidence that the DNA deamination activity of AID is dissociated from its physiological functions, i.e. CSR and SHM (Shivarov et al., 2008). All together, these findings encouraged us to assess the mutagenic activity of the AID-His 130Pro mutant using an SHM artificial substrate. While Jurkat cell line not expressing AID showed no fluorescent cells after pEM7TAG182 transfection, constitutive expression of AID-wt restored fluorescence in transfected Jurkat cells by $8 \%( \pm 3 \%)$. This reflects AID mutagenic activity that reverted EGFP nonsense mutation (TAG182). Indeed, ectopic AID expression in non-B cells promotes SHM in highly transcribed genes but also in artificial substrates. Accordingly, expression of AID-cofactors, required for SHM, are induced in B or non-B cells (Yoshikawa et al., 2002). Moreover, Rougeon and collaborators have demonstrated that SHM of transient transfected substrates is absolutely dependent on AID activity (Jovanic et al., 2008). In line with this finding, the AID-His56Tyr mutant was unable to restore fluorescence in pEM7-TAG182 transfected Jurkat cells. The p.(His56Tyr) 
mutation alters catalytic $\mathrm{Zn}^{2+}$ ion coordination in AID active center abolishing both CSR and SHM mechanisms (Durandy et al., 2006; Mu et al., 2012; Ta et al., 2003). In addition, the involvement of AID catalytic activity in the protein expression stability explains the very low amounts of AID-His56Tyr mutant protein observed by western blot analysis (Le and Maizels, 2015). Therefore, we conclude that the SHM vector based assay in Jurkat cell line is efficient to assess mutagenic activity of mutant AID proteins. Herein, we showed that AID-His130Pro mutant is able to revert the EGFP nonsense mutation as $5 \%( \pm 2 \%)$ of fluorescent cells were generated after pEM7TAG182 transfection. This suggests that the His130Pro mutation does not affect drastically AID mutagenic activity and that induced-somatic mutations may be retained at least on an artificial substrate. In the literature, only one AID mutation, located in APOBEC like domain, was reported to have variable SHM activity (Mahdaviani et al., 2012; Zhu et al., 2003). The others were either altering SHM or were not assessed. In parallel, all mutations in the APOBEC like domain were shown to affect CSR and cause HIGM2 syndrome (Mahdaviani et al., 2012; Ouadani et al., 2015).

AID initiates CSR and SHM by deaminating Cytosines to Uracils within the S and V regions respectively. In CSR, the $\mathrm{U}: \mathrm{G}$ mismatch is processed into double strand breaks followed by non-homologous end joining processing (Xu et al., 2012). In SHM, replication of the $\mathrm{U}: \mathrm{G}$ mismatch generates $\mathrm{C}$ to $\mathrm{T}$ mutations, whereas DNA repair mechanisms; i.e., Base excision repair and mismatch repair, generates mutations at G:C and A:T pairs (Peled et al., 2008).

On the other hand, AICDA mutations studies as well as biochemical studies of proteinprotein and RNA-protein binding showed that both CSR or SHM also depend on the interaction of AID with specific cofactors through different domains (Aida et al., 2013; 
Basu et al., 2011; Chaudhuri et al., 2004; Eid et al., 2014; Ganesh et al., 2011; Hu et al., 2013; Jeevan-Raj et al., 2011; Kanehiro et al., 2012; Lam et al., 2013; Nowak et al., 2011; Okazaki et al., 2011; Pavri et al., 2010). Regarding our data, they suggest that the AID-His130Pro mutant is able to catalyze $\mathrm{C}$ to $\mathrm{U}$ deamination required for SHM. Consequently, the resulting CSR deficiency is unlikely to be due to a defect in AID catalytic activity. Predictions based on the extensive homology between APOBEC family members revealed that the His 130 residue is localized in the $\alpha$-helix 4 of AID (Prochnow et al., 2007). The substitution of a histidine by a proline would then probably affect the structure of the protein (Richardson, 1981). However, it was demonstrated that His130 is not involved in the catalytic pocket of AID (King et al., 2015). In addition, a recent crystal structure of a soluble AID variant showed that the $\alpha$-helix 4 is part of a hydrophobic pocket capped by residues of loop 7 (Pham et al., 2016). These findings suggest that the probably altered structural integrity, caused by the His130Pro mutation, would not affect DNA deamination activity, whereas it could prevent a potential interaction of the enzyme with specific cofactors leading to CSR deficiency. Indeed, the His130 residue is located in a putative RNA-binding motif defined by amino acids 130 to 138 which are required for the binding of AID to G-quadruplex-RNA structures formed by $\mathrm{S}$ region transcripts. The formed AID-RNA complex directs the enzyme to S-DNA regions which is indispensable for CSR achievement (Zheng et al., 2015). Consistent with our results, the Gly133Val mutation, identified in two other HIGM2 patients (Mahdaviani et al., 2012) impairs the AID-RNA interaction but retains AID catalytic activity (Zheng et al., 2015). Furthermore, the AID-Gly133Val mutant was shown, recently, to be unable to interact with specific heterogeneous nuclear ribonucleoproteins (hnRNP) as well as mRNA-binding protein crucial for the CSR- 
recombination step. However, it binds to hnRNP K involved in DNA cleavage step required for both CSR and SHM (Yousif et al., 2014).

\section{Conclusion}

In summary, besides keeping the catalytic site of the protein intact, the His130Pro mutation allows the enzyme to retain its mutagenic activity, at least on an SHM artificial substrate. This mutation would prevent the interaction of AID with specific cofactors required for CSR but not for SHM. Thus, there would be no contradiction between maintaining the catalytic and mutagenic activity of AID and the presence of function defects at the genomic level. 


\section{Acknowledgements}

The authors declare that they have no competing interests.

This work was supported by the Ministry of Health (Tunisia). 


\section{References}

Adzhubei, I.A., Schmidt, S., Peshkin, L., Ramensky, V.E., Gerasimova, A., Bork, P., Kondrashov, A.S., Sunyaev, S.R., 2010. A method and server for predicting damaging missense mutations. Nat. Methods 7, 248-9. doi:10.1038/nmeth0410-248

Aida, M., Hamad, N., Stanlie, A., Begum, N.A., Honjo, T., 2013. Accumulation of the FACT complex, as well as histone H3.3, serves as a target marker for somatic hypermutation. Proc. Natl. Acad. Sci. U. S. A. 110, 7784-9. doi:10.1073/pnas.1305859110

Basu, U., Meng, F.-L., Keim, C., Grinstein, V., Pefanis, E., Eccleston, J., Zhang, T., Myers, D., Wasserman, C.R., Wesemann, D.R., Januszyk, K., Gregory, R.I., Deng, H., Lima, C.D., Alt, F.W., 2011. The RNA exosome targets the AID cytidine deaminase to both strands of transcribed duplex DNA substrates. Cell 144, 353-63. doi:10.1016/j.cell.2011.01.001

Caratão, N., Cortesão, C.S., Reis, P.H., Freitas, R.F., Jacob, C.M.A., Pastorino, A.C., CarneiroSampaio, M., Barreto, V.M., 2013. A novel activation-induced cytidine deaminase (AID) mutation in Brazilian patients with hyper-IgM type 2 syndrome. Clin. Immunol. 148, 27986. doi:10.1016/j.clim.2013.05.017

Chaudhuri, J., Khuong, C., Alt, F.W., 2004. Replication protein A interacts with AID to promote deamination of somatic hypermutation targets. Nature 430, 992-998.

doi:10.1038/nature02821

Crouch, E.E., Li, Z., Takizawa, M., Fichtner-Feigl, S., Gourzi, P., Montaño, C., Feigenbaum, L., Wilson, P., Janz, S., Papavasiliou, F.N., Casellas, R., 2007. Regulation of AID expression in the immune response. J. Exp. Med. 204, 1145-56. doi:10.1084/jem.20061952

Durandy, A., Kracker, S., Fischer, A., 2013. Primary antibody deficiencies. Nat. Rev. Immunol. 13, 519-33. doi:10.1038/nri3466 
Durandy, A., Peron, S., Taubenheim, N., Fischer, A., 2006. Activation-induced cytidine deaminase: structure-function relationship as based on the study of mutants. Hum. Mutat. 27, 1185-91. doi:10.1002/humu.20414

Eid, M.M.A., Maeda, K., Almofty, S.A., Singh, S.K., Shimoda, M., Sakaguchi, N., 2014. GANP regulates the choice of DNA repair pathway by DNA-PKcs interaction in AID-dependent IgV region diversification. J. Immunol. 192, 5529-39. doi:10.4049/jimmunol.1400021

Ganesh, K., Adam, S., Taylor, B., Simpson, P., Rada, C., Neuberger, M., 2011. CTNNBL1 is a novel nuclear localization sequence-binding protein that recognizes RNA-splicing factors CDC5L and Prp31. J. Biol. Chem. 286, 17091-102. doi:10.1074/jbc.M110.208769

Hu, Y., Ericsson, I., Torseth, K., Methot, S.P., Sundheim, O., Liabakk, N.B., Slupphaug, G., Di Noia, J.M., Krokan, H.E., Kavli, B., 2013. A combined nuclear and nucleolar localization motif in activation-induced cytidine deaminase (AID) controls immunoglobulin class switching. J. Mol. Biol. 425, 424-43. doi:10.1016/j.jmb.2012.11.026

Imai, K., Zhu, Y., Revy, P., Morio, T., Mizutani, S., Fischer, A., Nonoyama, S., Durandy, A., 2005. Analysis of class switch recombination and somatic hypermutation in patients affected with autosomal dominant hyper-IgM syndrome type 2. Clin. Immunol. 115, 277-85. doi:10.1016/j.clim.2005.02.003

Ito, S., Nagaoka, H., Shinkura, R., Begum, N., Muramatsu, M., Nakata, M., Honjo, T., 2004. Activation-induced cytidine deaminase shuttles between nucleus and cytoplasm like apolipoprotein B mRNA editing catalytic polypeptide 1. Proc. Natl. Acad. Sci. U. S. A. 101, 1975-80. doi:10.1073/pnas.0307335101

Jeevan-Raj, B.P., Robert, I., Heyer, V., Page, A., Wang, J.H., Cammas, F., Alt, F.W., Losson, R., Reina-San-Martin, B., 2011. Epigenetic tethering of AID to the donor switch region during immunoglobulin class switch recombination. J. Exp. Med. 208, 1649-60. 
doi:10.1084/jem.20110118

Jovanic, T., Roche, B., Attal-Bonnefoy, G., Leclercq, O., Rougeon, F., 2008. Ectopic expression of AID in a non-B cell line triggers A:T and G:C point mutations in non-replicating episomal vectors. PLoS One 3, e1480. doi:10.1371/journal.pone.0001480

Kanehiro, Y., Todo, K., Negishi, M., Fukuoka, J., Gan, W., Hikasa, T., Kaga, Y., Takemoto, M., Magari, M., Li, X., Manley, J.L., Ohmori, H., Kanayama, N., 2012. Activation-induced cytidine deaminase (AID)-dependent somatic hypermutation requires a splice isoform of the serine/arginine-rich (SR) protein SRSF1. Proc. Natl. Acad. Sci. 109, 1216-1221. doi:10.1073/pnas.1120368109

King, J.J., Manuel, C.A., Barrett, C. V, Raber, S., Lucas, H., Sutter, P., Larijani, M., 2015. Catalytic pocket inaccessibility of activation-induced cytidine deaminase is a safeguard against excessive mutagenic activity. Structure 23, 615-27. doi:10.1016/j.str.2015.01.016

Kohli, R.M., Abrams, S.R., Gajula, K.S., Maul, R.W., Gearhart, P.J., Stivers, J.T., 2009. A portable hot spot recognition loop transfers sequence preferences from APOBEC family members to activation-induced cytidine deaminase. J. Biol. Chem. 284, 22898-904. doi:10.1074/jbc.M109.025536

Kracker, S., Imai, K., Gardès, P., Ochs, H.D., Fischer, A., Durandy, A.H., 2010. Impaired induction of DNA lesions during immunoglobulin class-switch recombination in humans influences end-joining repair. Proc. Natl. Acad. Sci. U. S. A. 107, 22225-30. doi:10.1073/pnas.1012591108

Kumar, P., Henikoff, S., Ng, P.C., 2009. Predicting the effects of coding non-synonymous variants on protein function using the SIFT algorithm. Nat. Protoc. 4, 1073-81. doi:10.1038/nprot.2009.86

Lam, T., Thomas, L.M., White, C.A., Li, G., Pone, E.J., Xu, Z., Casali, P., 2013. Scaffold 
functions of 14-3-3 adaptors in B cell immunoglobulin class switch DNA recombination. PLoS One 8, e80414. doi:10.1371/journal.pone.0080414

Le, Q., Maizels, N., 2015. Cell Cycle Regulates Nuclear Stability of AID and Determines the Cellular Response to AID. PLoS Genet. 11, e1005411. doi:10.1371/journal.pgen.1005411

Mahdaviani, S.A., Hirbod-Mobarakeh, A., Wang, N., Aghamohammadi, A., Hammarström, L., Masjedi, M.R., Pan-Hammarström, Q., Rezaei, N., 2012. Novel mutation of the activationinduced cytidine deaminase gene in a Tajik family: special review on hyperimmunoglobulin M syndrome. Expert Rev. Clin. Immunol. 8, 539-46. doi:10.1586/eci.12.46

Mondal, S., Begum, N.A., Hu, W., Honjo, T., 2016. Functional requirements of AID’s higher order structures and their interaction with RNA-binding proteins. Proc. Natl. Acad. Sci. U. S. A. doi:10.1073/pnas.1601678113

Mu, Y., Prochnow, C., Pham, P., Chen, X.S., Goodman, M.F., 2012. A structural basis for the biochemical behavior of activation-induced deoxycytidine deaminase class-switch recombination-defective hyper-IgM-2 mutants. J. Biol. Chem. 287, 28007-16. doi:10.1074/jbc.M112.370189

Muramatsu, M., Kinoshita, K., Fagarasan, S., Yamada, S., Shinkai, Y., Honjo, T., 2000. Class switch recombination and hypermutation require activation-induced cytidine deaminase (AID), a potential RNA editing enzyme. Cell 102, 553-63.

Muto, T., Muramatsu, M., Taniwaki, M., Kinoshita, K., Honjo, T., 2000. Isolation, tissue distribution, and chromosomal localization of the human activation-induced cytidine deaminase (AID) gene. Genomics 68, 85-8. doi:10.1006/geno.2000.6268

Nowak, U., Matthews, A.J., Zheng, S., Chaudhuri, J., 2011. The splicing regulator PTBP2 interacts with the cytidine deaminase AID and promotes binding of AID to switch-region DNA. Nat. Immunol. 12, 160-6. doi:10.1038/ni.1977 
Okazaki, I., Okawa, K., Kobayashi, M., Yoshikawa, K., Kawamoto, S., Nagaoka, H., Shinkura, R., Kitawaki, Y., Taniguchi, H., Natsume, T., Iemura, S.-I., Honjo, T., 2011. Histone chaperone Spt6 is required for class switch recombination but not somatic hypermutation. Proc. Natl. Acad. Sci. U. S. A. 108, 7920-5. doi:10.1073/pnas.1104423108

Ouadani, H., Ben-Mustapha, I., Ben-Ali, M., Ben-Khemis, L., Larguèche, B., Boussoffara, R., Maalej, S., Fetni, I., Hassayoun, S., Mahfoudh, A., Mellouli, F., Yalaoui, S., Masmoudi, H., Bejaoui, M., Barbouche, M.-R., 2015. Novel and recurrent AID mutations underlie prevalent autosomal recessive form of HIGM in consanguineous patients. Immunogenetics. doi:10.1007/s00251-015-0878-6

Patenaude, A.-M., Orthwein, A., Hu, Y., Campo, V.A., Kavli, B., Buschiazzo, A., Di Noia, J.M., 2009. Active nuclear import and cytoplasmic retention of activation-induced deaminase. Nat. Struct. Mol. Biol. 16, 517-27. doi:10.1038/nsmb.1598

Patiroglu, T., Akar, H.H., van der Burg, M., Unal, E., 2015. Autosomal recessive hyper IgM syndrome associated with activation-induced cytidine deaminase gene in three Turkish siblings presented with tuberculosis lymphadenitis - Case report. Acta Microbiol. Immunol. Hung. 62, 267-74. doi:10.1556/030.62.2015.3.4

Pavri, R., Gazumyan, A., Jankovic, M., Di Virgilio, M., Klein, I., Ansarah-Sobrinho, C., Resch, W., Yamane, A., Reina San-Martin, B., Barreto, V., Nieland, T.J., Root, D.E., Casellas, R., Nussenzweig, M.C., 2010. Activation-induced cytidine deaminase targets DNA at sites of RNA polymerase II stalling by interaction with Spt5. Cell 143, 122-33. doi:10.1016/j.cell.2010.09.017

Peled, J.U., Kuang, F.L., Iglesias-Ussel, M.D., Roa, S., Kalis, S.L., Goodman, M.F., Scharff, M.D., 2008. The biochemistry of somatic hypermutation. Annu. Rev. Immunol. 26, 481511. doi:10.1146/annurev.immunol.26.021607.090236 
Pham, P., Afif, S.A., Shimoda, M., Maeda, K., Sakaguchi, N., Pedersen, L.C., Goodman, M.F., 2016. Structural analysis of the activation-induced deoxycytidine deaminase required in immunoglobulin diversification. DNA Repair (Amst). 43, 48-56.

doi:10.1016/j.dnarep.2016.05.029

Prochnow, C., Bransteitter, R., Klein, M.G., Goodman, M.F., Chen, X.S., 2007. The APOBEC-2 crystal structure and functional implications for the deaminase AID. Nature 445, 447-51. doi:10.1038/nature05492

Revy, P., Muto, T., Levy, Y., Geissmann, F., Plebani, A., Sanal, O., Catalan, N., Forveille, M., Dufourcq-Labelouse, R., Gennery, A., Tezcan, I., Ersoy, F., Kayserili, H., Ugazio, A.G., Brousse, N., Muramatsu, M., Notarangelo, L.D., Kinoshita, K., Honjo, T., Fischer, A., Durandy, A., 2000. Activation-induced cytidine deaminase (AID) deficiency causes the autosomal recessive form of the Hyper-IgM syndrome (HIGM2). Cell 102, 565-75.

Richardson, J.S., 1981. The anatomy and taxonomy of protein structure. Adv. Protein Chem. 34, $167-339$.

Sabouri, S., Kobayashi, M., Begum, N.A., Xu, J., Hirota, K., Honjo, T., 2014. C-terminal region of activation-induced cytidine deaminase (AID) is required for efficient class switch recombination and gene conversion. Proc. Natl. Acad. Sci. U. S. A. 111, 2253-8. doi:10.1073/pnas.1324057111

Shinkura, R., Ito, S., Begum, N.A., Nagaoka, H., Muramatsu, M., Kinoshita, K., Sakakibara, Y., Hijikata, H., Honjo, T., 2004. Separate domains of AID are required for somatic hypermutation and class-switch recombination. Nat. Immunol. 5, 707-712. doi:10.1038/ni1086

Shivarov, V., Shinkura, R., Honjo, T., 2008. Dissociation of in vitro DNA deamination activity and physiological functions of AID mutants. Proc. Natl. Acad. Sci. U. S. A. 105, 15866-71. 
doi:10.1073/pnas.0806641105

Ta, V.-T., Nagaoka, H., Catalan, N., Durandy, A., Fischer, A., Imai, K., Nonoyama, S., Tashiro, J., Ikegawa, M., Ito, S., Kinoshita, K., Muramatsu, M., Honjo, T., 2003. AID mutant analyses indicate requirement for class-switch-specific cofactors. Nat. Immunol. 4, 843-8. doi:10.1038/ni964

Trotta, L., Hautala, T., Hämäläinen, S., Syrjänen, J., Viskari, H., Almusa, H., Lepisto, M., Kaustio, M., Porkka, K., Palotie, A., Seppänen, M., Saarela, J., 2016. Enrichment of rare variants in population isolates: single AICDA mutation responsible for hyper-IgM syndrome type 2 in Finland. Eur. J. Hum. Genet. doi:10.1038/ejhg.2016.37

Wedekind, J.E., Dance, G.S.C., Sowden, M.P., Smith, H.C., 2003. Messenger RNA editing in mammals: new members of the APOBEC family seeking roles in the family business. Trends Genet. 19, 207-16. doi:10.1016/S0168-9525(03)00054-4

Xu, Z., Zan, H., Pone, E.J., Mai, T., Casali, P., 2012. Immunoglobulin class-switch DNA recombination: induction, targeting and beyond. Nat. Rev. Immunol. 12, 517-31. doi:10.1038/nri3216

Yoshikawa, K., Okazaki, I.-M., Eto, T., Kinoshita, K., Muramatsu, M., Nagaoka, H., Honjo, T., 2002. AID enzyme-induced hypermutation in an actively transcribed gene in fibroblasts. Science 296, 2033-6. doi:10.1126/science.1071556

Yousif, A.S., Stanlie, A., Mondal, S., Honjo, T., Begum, N.A., 2014. Differential regulation of Sregion hypermutation and class-switch recombination by noncanonical functions of uracil DNA glycosylase. Proc. Natl. Acad. Sci. U. S. A. 111, E1016-24. doi:10.1073/pnas.1402391111

Zan, H., Casali, P., 2013. Regulation of Aicda expression and AID activity. Autoimmunity 46, 83-101. doi:10.3109/08916934.2012.749244 
Zheng, S., Vuong, B.Q., Vaidyanathan, B., Lin, J.-Y., Huang, F.-T., Chaudhuri, J., 2015. Noncoding RNA Generated following Lariat Debranching Mediates Targeting of AID to DNA. Cell 161, 762-773. doi:10.1016/j.cell.2015.03.020

Zhu, Y., Nonoyama, S., Morio, T., Muramatsu, M., Honjo, T., Mizutani, S., 2003. Type two hyper-IgM syndrome caused by mutation in activation-induced cytidine deaminase. J. Med. Dent. Sci. 50, 41-6.

\footnotetext{
1 Janelia Farm Research Campus, Howard Hughes Medical Institute, Ashburn, Virginia, United States of America. ${ }^{2}$ Laboratory of Clinical Infectious Diseases, National Institute of Allergy and Infectious Diseases, National Institutes of Health, Bethesda, MD, USA.

${ }^{3}$ College of Postgraduate Studies, Arabian Gulf University, Manama, Bahrain.
} 
Figure 1: AID expression assessment. a) AICDA transcription in stable Jurkat cell lines. AID transcripts were amplified from by RT-PCR from Jurkat, Jurkat AID, Jurkat AIDHis56Tyr and Jurkat AID-His130Pro total RNA. T- : negatif $\left(\mathrm{H}_{2} \mathrm{O}\right)$ PCR control. b) Western Blot of AID protein. AID proteins from a healthy individual and an HIGM2 patient (AID-His130Pro) EBV LCLs lysates (lanes I and II) and stable Jurkat cells lysates (lanes III, IV, V and VI) were revealed using a rabbit monoclonal anti AID. I: healthy control (AID), II: HIGM2 patient (AID-His130Pro), III: Jurkat, IV: Jurkat AID, V: Jurkat AIDHis56Tyr and VI: Jurkat AID-His130Pro. $\beta$-actin was used as a loading control.

a)

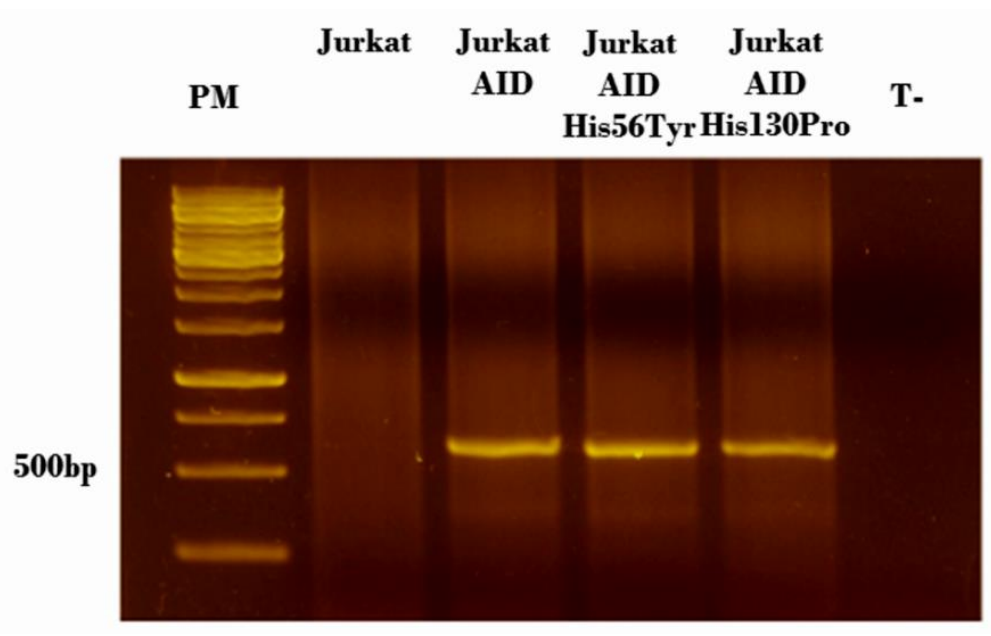

b)

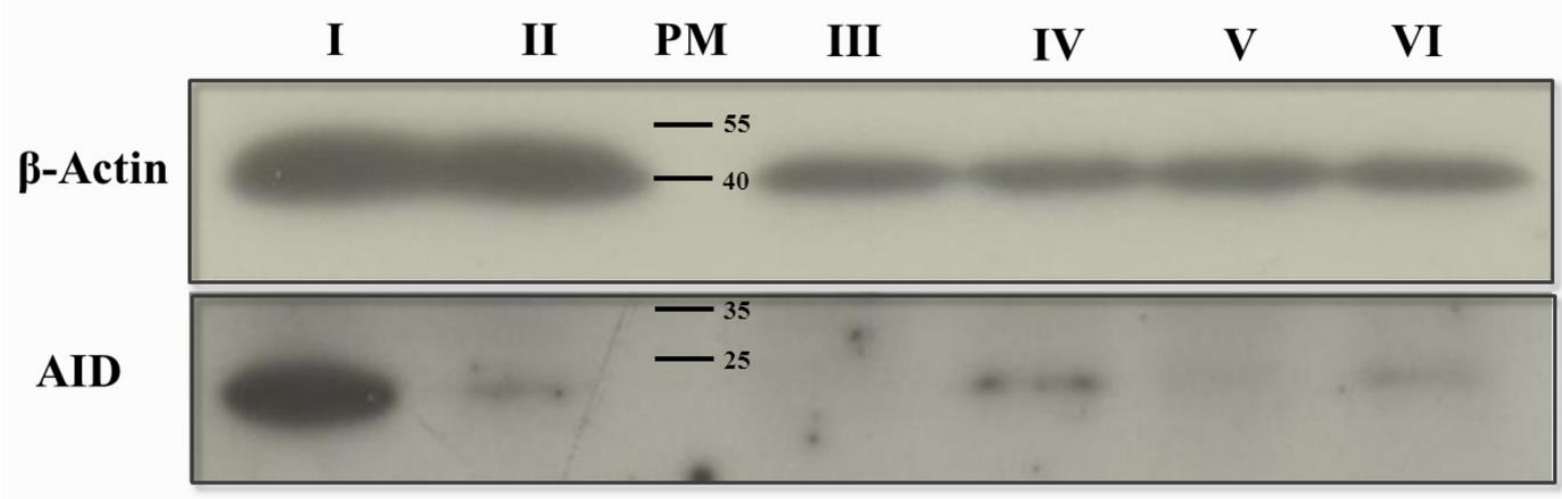


Figure 2: Assessment of Jurkat cell lines transfection efficiency by flow cytometry. Jurkat, Jurkat AID, Jurkat AID-His56Tyr and Jurkat AID-His130Pro stable cell lines were transfected by pEM7EGFP. Analyzes were done 24 hours after transfection using FlowJo V.10 software.
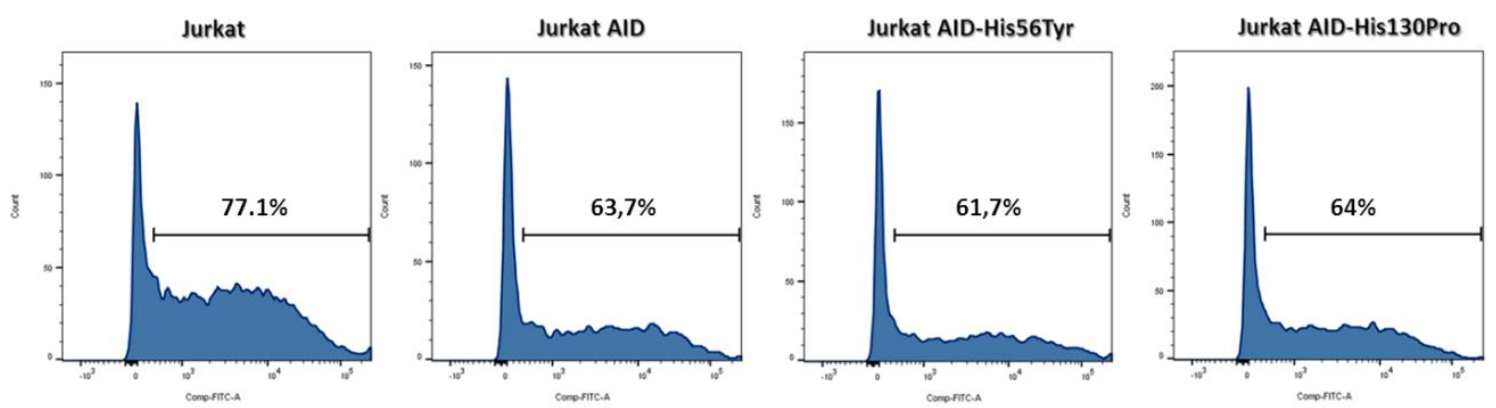

Figure 3: Assessment of reverted fluorescent cells by flow cytometry. Jurkat, Jurkat AID, Jurkat AIDHis56Tyr and Jurkat AID-His130Pro stable cell lines were transfected by pEM7-TAG182. Analyzes were done 24 hours after transfection using FlowJo V.10 software. I and II are distinct experiences.
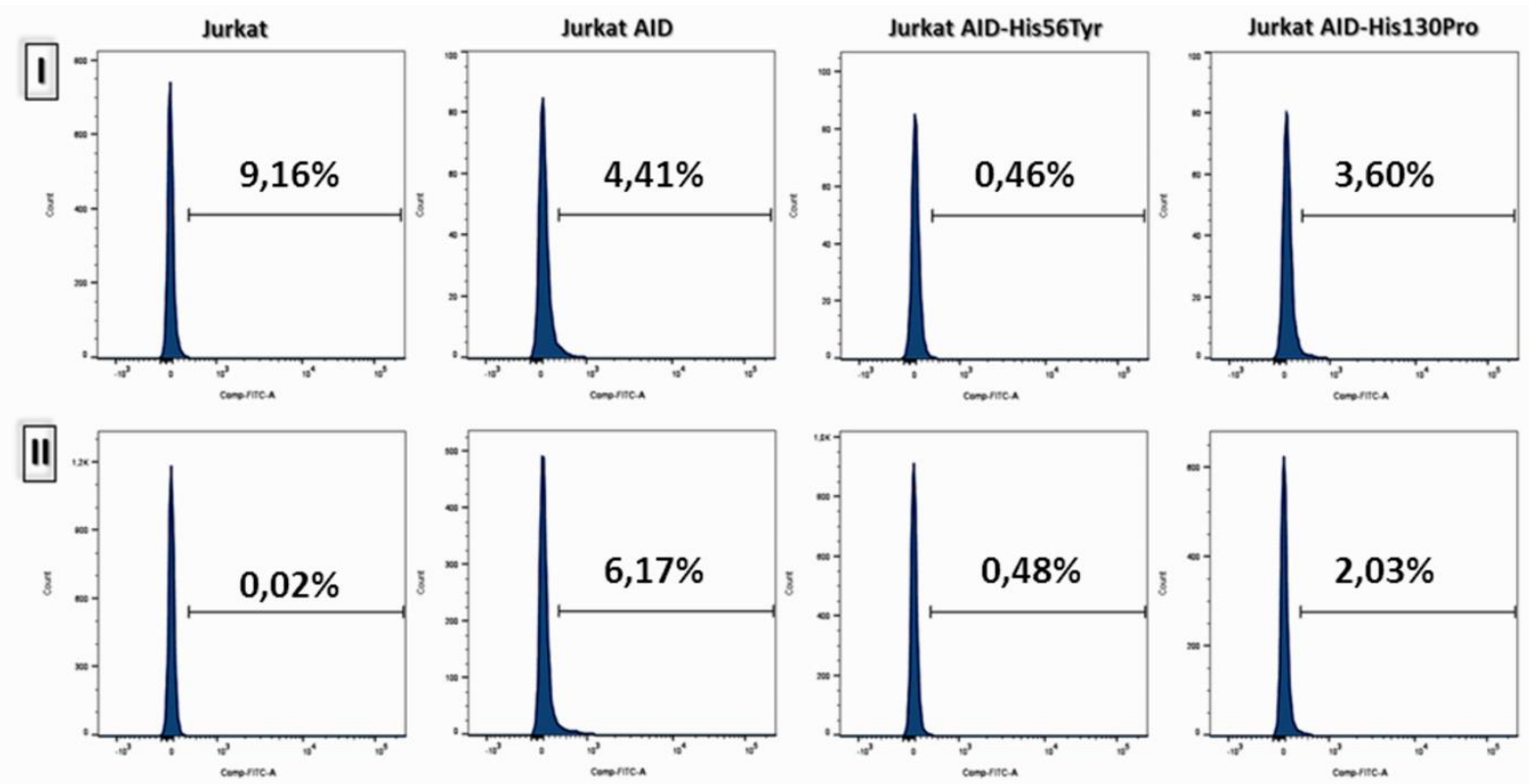
Figure 4: AID mutagenesis activity. The mutagenesis activity is represented by the reversion rate (\% of reverted fluorescent cells) relative to the transfection efficiency. This data represent the average values of two independent experiments. Confidences ranges are represented by error bars.

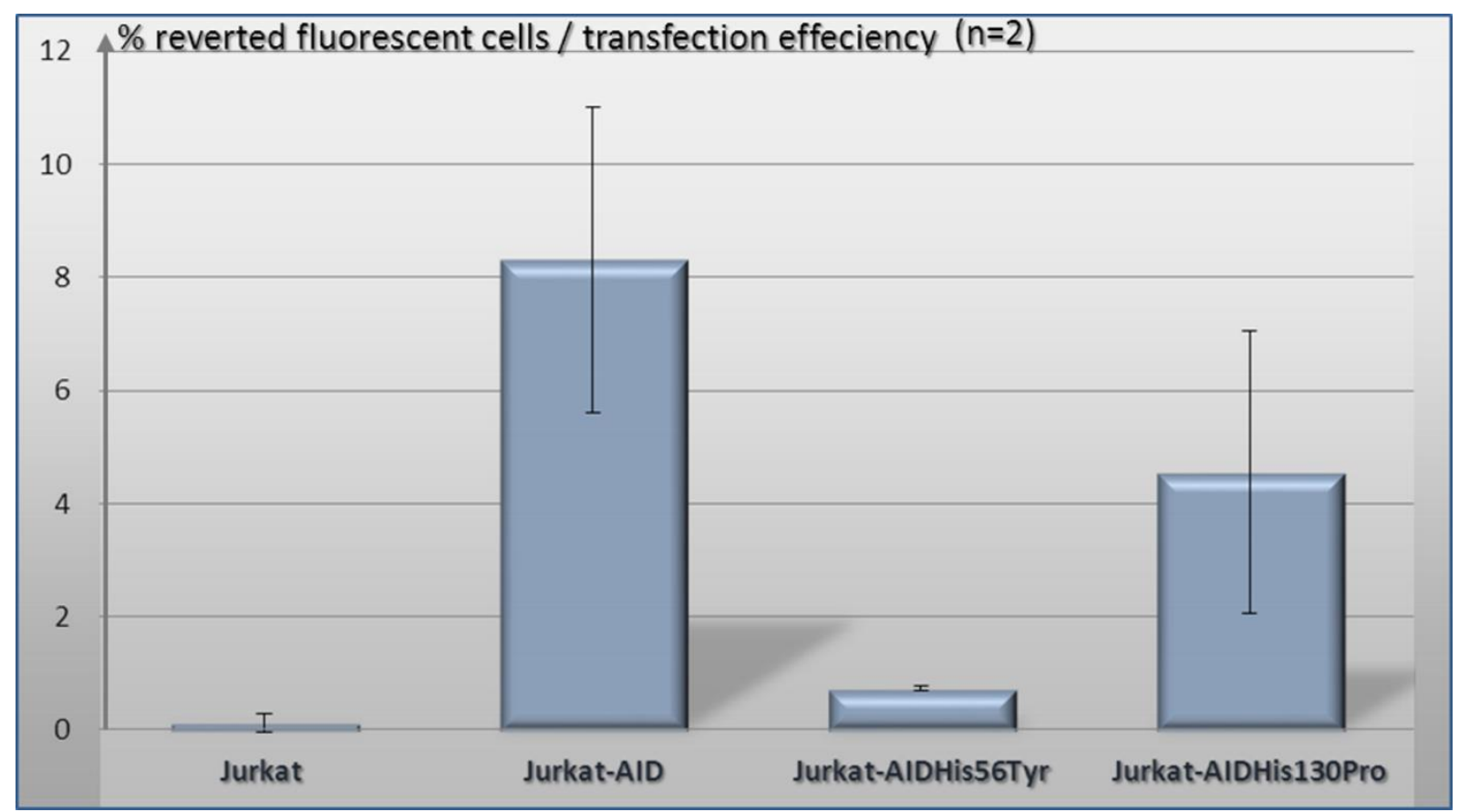

